823

\section{EPIDEMIOLOGICAL CHARACTERISTICS OF DOWN SYNDROME IN THE REGION OF WESTERN HERZEGOVINA}

D. Šumanović--Glamuzina ${ }^{1}$, T. Božić ${ }^{1}$, Ž. Bilinovac ${ }^{1}$, I. Letica ${ }^{1}$, M. Vukojević ${ }^{2}$

1 University Hospital Mostar, ${ }^{2}$ School of Medicine University of Mostar, Mostar, Bosnia-Herzegovina

During the 15 years period (1994-2008), 46 children with Down syndrome (DS) (31 boys and 15 girls) have been registered at the Neonatology Department of the University Hospital Mostar (Bosnia and Herzegovina). The average prevelence of DS for the region of Western Herzegovina (WH) (217 944 inhabitants) is 1,5 per 1,000 newborns in that period.

DS has been proved by means citogenetic analyses in 38 children. In 36 there was a regular type of trisomy $21(94.7 \%)$ and in 2 the translocation type of DS was found.

33 parental couples $(71.7 \%)$ were over 35 years of age. Early amniocentesis due to the parent's age was made in two cases only, with positive dignosis. Usually, parents are refusing prenatal diagnosis due to religous reasons.

All children with DS have had five or more easily recognized minor malformations present in variable combinations. 28/46 had major malformations and three children have had mild forms of conatal hypothyreosis. Seven children (15.2\%) died in neonatal or early infant period due to sepsis and complex cardiovascular anomalies. Considering evidently present major malformations and accompaning complications, DS remains very important issue for routine pediatric clinical practice.

\section{4}

\section{CONGENITAL HAND DEFORMITIES AND ASSOCIATED SYNDROMES}

\author{
J.E. $\operatorname{Tan}^{1}$, J.B. O Sullivan², K. Cronin² \\ ${ }^{1}$ Department of Plastic and Reconstructive \\ Surgery, Children's University Hospital, \\ ${ }^{2}$ Department of Plastics and Reconstructive \\ Surgery, Childrens University Hospital, \\ Dublin, Ireland
}

Background: Congenital Hand Deformity is an upper limb abnormality which present at the time of birth. Only $5 \%$ of congenital hand deformities occur as part of a recognized syndrome.

Objective: To retrospectively review all congenital hand deformities treated in Children University Hospital from January 2003 to October 2009 and correlate with syndromes associated.

Methods: Getting the list of hand surgeries from theatre. Retrieve charts from Chart Library. Compiled and enter data in Microsoft Excel.

Results: $20 \%$ patients have an associated syndrome. $29.3 \%$ presented with polydactyly. 7 of them are associated with syndromes. 59 out of 317 has syndactyly and $15 \%$ are associated with Apert Syndrome. 70 out of 317 are associated with radial ray abnormality and $24 \%$ has a syndromic background. None of the radial ray abnormality are associated with fanconi anaemia. 45 out of 317 presented with multiple ulnar deformities and 3 are associated with syndromes. 38 out of 317 presented with symbrachydactyly and $21 \%$ are associated with various syndrome. 10 out of 317 are associated with multiple hand deformity and mostly they are associated with amniotic band syndrome. There are 2 patients that present with macrodactyly, however they are no medical association noted.

Discussion: In conclusion, $19.9 \%$ of the patients has associated medical background. None of them has proven Fanconi Anaemia manifestation. Fanconi Anaemia is commonly associated with radial aplasia.

Hence in the future, we will retrospectively screen all patient that has radial ray abnormality and prospectively screen them for fanconi anemia screening test.

825

\section{TRISOMY 13 WITH ANORECTAL MALFORMATION:AN ASSOCIATION OR AN INCIDENTAL FINDING}

\author{
P.K. Arora ${ }^{1}$, N. Aladangady ${ }^{2}$
}

${ }^{1}$ Neonates, Homerton University Hospital NHS Trust, ${ }^{2}$ Neonates, Barts and the London School of Medicine and Dentistry, London, UK

This report describes an infant with trisomy 13 associated with imperforate anus. Clinical features of patau syndrome described in standard texts do not include imperforate anus or anorectal malformations (ARM). In our search of the English 
literature only four cases of trisomy 13 have being associated with imperforate anus: a de novo mosaicism 46,XX,t(13q13q)/46,XX,-13,+r(13), a partial trisomy of chromosome $13 q$ and $20 p$ and trisomy 13 with Robertsonian translocation of extra chromosome 13 to chromosome 14. The fourth case of trisomy 13 has been mentioned by Cho et al in 2001 in which the form of trisomy has not been described.

This baby girl was born at term by emergency caesarian section for fetal tachycardia to non consanguineous parents. On initial examination the she was noted to have overlapping fingers, widely spacednipples, ulnar deviation of hands, microphthalmia with ectropion of the right eye. Systemic examination revealed imperforate anus and a systolic murmur. The baby was transferred to a surgical unit and had a colostomy on day one of life under general anaesthesia. She was discharged home on day 21 of life.

Out of the four cases of trisomy 13 described so far with imperforate anus all have been either mosaics or translocations. Our baby is unique as she is possibly the only reported child with Patau syndrome $47 X X+13$ (pure non disjunction and not mosaic/ translocation) to have anorectal malformation. It is still questionable whether imperforate anus is a true association of trisomy 13 or just a co-incidental occurence.

\section{6}

\section{MOLECULAR TOOL IN THE DIAGNOSIS AND ANTENATAL DIAGNOSIS IN GLYCOGEN STORAGE DISEASE TYPE IA IN TUNISIAN PATIENTS}

\author{
E. Barkaoui, C. Charfeddine, W. Cherif, \\ S. Abdelhak \\ Pasteur Institut, Tunis, Tunisia
}

Introduction: Glycogen storage disease type la is an autosomal recessive disorder of glycogen metabolism caused by glucose-6-phosphatase deficiency. It is characterized by short stature, hepatomegaly, hypoglycaemia, hyperuricemia, and hyperlactacidemia. The gene G6PC was cloned by lei et al in 1993.

Patients and methods: A study of the molecular basis of GSD type la was carried out in 8 Tunisian patients from 7 unrelated families. For five patients the diagnosis was performed by enzyme assay in fresh liver. To order to identify the mutations segregating with Tunisian families, exons from G6PC gene were amplified for genomic DNA of patients and directly sequenced.

Results: Five patients had the R83C recurrent mutation and one had the R170Q. Both the tow mutations are known to abolish G-6-Pase activity and therefore are responsible for severe form of GSD type la. R83C and R170Q are relatively frequent among Turkish patients.

Conclusion: High prevalence of the R83C mutation might be due not only to a hot spot mutation but also is probably due to population migration. As most of the Tunisian patients bare the R83C mutation, direct screening of this mutation will be a convenient alternative to the enzyme assay in fresh liver biopsy specimen for the diagnosis of GSD type la. Since this work all patients (22 cases) suspected of GSD type la were diagnosticated by molecular biology.

\section{7}

\section{SLC25A19 MUTATION IS A NOVEL CAUSE OF NEUROPATHY AND BILATERAL STRIATAL NECROSIS}

\author{
R. Spiegel ${ }^{1}$, A. Shaag ${ }^{2}$, S. Edvardson ${ }^{2}$, \\ H. Mandel ${ }^{3}$, S. Shalev ${ }^{4}$, Y. Horovitz ${ }^{1}$, O. Elpeleg ${ }^{2}$ \\ ${ }^{1}$ Pediatric Ward A, HaEmek Medical Center, \\ Afula, ${ }^{2}$ The Metabolic Disease Unit, Hadassah \\ - Hebrew University Medical Center, Jerusalem, \\ ${ }^{3}$ The Metabolic Unit, Mayer Medical Center, Haifa, \\ ${ }^{4}$ Genetic Institute, HaEmek Medical Center, \\ Afula, Israel
}

Acute basal ganglia necrosis in the pediatric age group is a neurological disorder characterized by symmetrical degeneration of the caudate nucleus, putamen, and occasionally the globus pallidus. The outcome is less favorable for the familial cases where the course is relentlessly progressive or punctuated by recurrent episodes with involvement of additional brain regions and significant residual neurological damage. The differential diagnosis of the latter group includes mitochondrial respiratory chain defects organic acid disorders, Wilson disease, juvenile Huntington's chorea, pantothenate kinaseassociated neurodegeneration, biotin responsive basal ganglia disease, and the striatal necrosis associated with NUP62 mutation.

In this study we report on four siblings from a consanguineous Arab Muslim family who suffered from recurrent episodes of flaccid paralysis and encephalopathy and chronic progressive 